\title{
Rhythmic Abilities of Children With Hearing Loss
}

\author{
Céline Hidalgo, ${ }^{1,2}$ Annabelle Zécri, ${ }^{1}$ Jacques Pesnot-Lerousseau, ${ }^{1}$ Eric Truy, ${ }^{3,4,5,6}$ \\ Stéphane Roman, ${ }^{1,7}$ Simone Falk, ${ }^{8,9}$ Simone Dalla Bella, ${ }^{9,10,11}$ and Daniele Schön ${ }^{1}$
}

\begin{abstract}
Objectives: Children with hearing loss (HL), in spite of early cochlear implantation, often struggle considerably with language acquisition. Previous research has shown a benefit of rhythmic training on linguistic skills in children with $\mathrm{HL}$, suggesting that improving rhythmic capacities could help attenuating language difficulties. However, little is known about general rhythmic skills of children with HL and how they relate to speech perception. The aim of this study is twofold: (1) to assess the abilities of children with HL in different rhythmic sensorimotor synchronization tasks compared to a normal-hearing control group and (2) to investigate a possible relation between sensorimotor synchronization abilities and speech perception abilities in children with $\mathrm{HL}$.
\end{abstract}

Design: A battery of sensorimotor synchronization tests with stimuli of varying acoustic and temporal complexity was used: a metronome, different musical excerpts, and complex rhythmic patterns. Synchronization abilities were assessed in 32 children (aged from 5 to 10 years) with a severe to profound HL mainly fitted with one or two cochlear implants $(n=28)$ or with hearing aids $(n=4)$. Working memory and sentence repetition abilities were also assessed. Performance was compared to an age-matched control group of 24 children with normal hearing. The comparison took into account variability in working memory capacities. For children with HL only, we computed linear regressions on speech, sensorimotor synchronization, and working memory abilities, including device-related variables such as onset of device use, type of device, and duration of use.

Results: Compared to the normal-hearing group, children with HL performed poorly in all sensorimotor synchronization tasks, but the effect size was greater for complex as compared to simple stimuli. Group differences in working memory did not explain this result. Linear regression analysis revealed that working memory, synchronization to complex rhythms performances, age, and duration of device use predicted the number of correct syllables produced in a sentence repetition task.

Conclusion: Despite early cochlear implantation or hearing aid use, hearing impairment affects the quality of temporal processing of acoustic stimuli in congenitally deaf children. This deficit seems to be more severe with stimuli of increasing rhythmic complexity highlighting a difficulty in structuring sounds according to a temporal hierarchy.

Key words: Acoustic structure complexity, Cochlear implants, Hearing Ioss, Rhythmic abilities, Sensorimotor synchronization, Temporal structure complexity.

(Ear \& Hearing 2020;XX;00-00)

${ }^{1}$ Aix Marseille University, Inserm, INS, Institut de Neurosciences des Systèmes, Marseille, France; ${ }^{2}$ Aix Marseille University, CNRS, LPL, Aix-en Provence, France; ${ }^{3}$ Service d'ORL et de Chirurgie cervico-faciale, Hôpital Edouard Herriot, CHU LYON, France; ${ }^{4}$ Inserm U1028, Lyon Neuroscience Research Center, Equipe IMPACT, Lyon, France; ${ }^{5}$ CNRS UMR5292, Lyon Neuroscience Research Center, Equipe IMPACT, Lyon, France; ${ }^{6}$ University Lyon 1, Lyon, France; ${ }^{7}$ Pediatric Otolaryngology Department, La Timone Children's Hospital (APHM), Marseille, France; ${ }^{8}$ Department of Linguistics and Translation, University of Montreal, Montreal, Canada; ${ }^{9}$ International Laboratory for Brain, Music, and Sound Research (BRAMS), Montreal Canada; ${ }^{10}$ Department of Psychology, University of Montreal, Montreal, Canada; and ${ }^{11}$ Centre for Research on Brain, Language and Music (CRBLM), Montreal, Canada.

Supplemental digital content is available for this article. Direct URL citations appear in the printed text and are provided in the HTML and text of this article on the journal's Web site (www.ear-hearing.com).

\section{INTRODUCTION}

Thanks to neonatal screening and early implantation, children with a cochlear implant (CI) can better decode speech and learn to speak in an oral environment, compared to peers wearing conventional hearing aids (Truy et al. 1998; Baldassari et al. 2009). However, while children with CI can achieve age-appropriate receptive vocabulary skills (Hayes et al. 2009), most of them achieve poorer vocabulary knowledge (Lund 2016) and lower verbal fluency than normal-hearing (NH) children (Wechsler-Kashi et al. 2014). Moreover, compared to their $\mathrm{NH}$ peers, children with CI perform poorer in the morphological and syntactical domain (Boons et al. 2013a) and in spoken narrative skills (Boons et al. 2013a, 2013b). Linguistic difficulties also persist in speech perception. Difficulties with prosody, such as discrimination of vocal emotions and processing of prosodic discourse markers, are quite common (Torppa et al. 2014; Holt et al. 2017; Jiam et al. 2017). Lower scores are also observed in reading skills (Johnson \& Goswami 2010; Geers et al. 2011), discrimination of phonologically similar words, grammatical processing (Caselli et al. 2012), but also in discursive and abstract reasoning (Bandursky \& Gałkowski 2004; Geers et al. 2011; see van Wieringen \& Wouters 2015 for a recent review of linguistics skills of children with CI). Finally, while the intelligibility of speech of children with $\mathrm{CI}$ can be good (Peng et al. 2004; Habib et al. 2010), speech production scores remain quite variable (Blamey et al. 2001). Globally, most children with $\mathrm{CI}$ have a lower speech intelligibility than their NH peers (Grandon et al. 2020).

Interestingly, the training of rhythmic skills seems to attenuate some of these verbal deficits in children with hearing loss (HL). Recently, we showed that children's capacity to temporally anticipate and adapt to a partner during verbal interaction was improved following a rhythm intervention (Hidalgo et al. 2017, 2019). Moreover, in general, rhythmic stimulation seems to facilitate different levels of language processing. For example, rhythmic priming enhances sentence perception, which in turn improves production abilities in children with HL (Cason et al. 2015). However, little is known about the general rhythmic abilities of children with HL. It is possible that the general rhythmic capacities of children with $\mathrm{HL}$ are poorer compared to NH children, pointing to general deficits in temporal processing caused by auditory impairment. It is also possible that the better their rhythmic skills the better they process finegrained temporal auditory stimuli (i.e., similarly to differences found between musicians and nonmusicians; see Rammsayer \& Altenmüller 2006; Chobert et al. 2011; Sares et al. 2018). Improving our knowledge of general rhythmic capacities of children with HL is important for understanding how sensorimotor and cognitive abilities, involved in rhythm and timing processing, interface with verbal abilities of children with HL. Indeed, in certain developmental speech and language disorders, rhythmic skills are tightly linked to verbal capacities. For 
example, poor performance in nonverbal sensorimotor synchronization tasks, such as tapping along the beat or rhythm reproduction, is linked to deficits in reading abilities and stuttering severity (Thomson \& Goswami 2008; Tierney \& Kraus 2013a; Flaugnacco et al. 2014; Falk et al. 2015). Rhythmic stimulation can also improve syntax processing in children with specific language impairment (Przybylski et al. 2013; Bedoin et al. 2016) and phonological awareness in children with dyslexia (Flaugnacco et al. 2015). Thus, gaining a better understanding of general rhythmic capacities of children with HL might help to devise interventions based on musical training that can engage children with HL in social musical activities, with benefits potentially extending to nonmusical domains (Phillips-Silver et al. 2015). Finally, knowledge of individual differences in rhythmic abilities in children with HL may allow to predict the success of a rhythm-based intervention, as found in certain diseases (e.g., Parkinson's disease, Dalla Bella et al. 2017a, 2018; Cochen De Cock et al. 2018).

Interestingly, when considering the neuroscientific literature on rhythm processing and brain plasticity, rhythmic training has been proposed to facilitate the processing of verbal sounds because it enhances the precision of auditory timing (Tierney \& Kraus 2014) and improves temporal prediction (Kotz \& Schwartze 2010). This facilitation effect is likely to be underpinned by the capacity of our neural system to adapt/predict the hierarchical organization of sound (Giraud \& Poeppel 2012; Peelle \& Davis 2012). The neural oscillatory activity of the auditory system entrains to event periodicities at different time scales, such as the syllable, but also recurrences or larger prosodic units like phrases (Luo \& Poeppel 2007; Bourguignon et al. 2013; Ding \& Simon 2014; Doelling et al. 2014). The dynamics of these complex oscillatory processes creates a scaffolding to support auditory perception and comprehension of hierarchical verbal structure (Ding et al. 2016; Kösem \& van Wassenhove 2017).

Here, we hypothesize that children with HL may have difficulties in perceiving the temporal hierarchical organization of sounds, which is essential for speech and language processing. Prenatal or perinatal HL may lead to deficits in auditory attention (Torppa et al. 2014) and particularly in attention to speech (Houston et al. 2003; Segal \& Kishon-Rabin 2011; Houston \& Bergeson 2014; Wang et al. 2018) also including prosodic cues (Holt et al. 2016). In the visual modality, children with CI struggle to organize sequential events into a hierarchical structure (Conway et al. 2011). Notably, these sequencing skills correlate with speech and language deficits such as sentence repetition. In the auditory modality, some studies show impaired discrimination of auditory rhythmic sequences in children with CI relative to NH children (Stabej et al. 2012; Innes-Brown et al. 2013; Good et al. 2017; Polonenko et al. 2017). Given the paucity of recent studies on rhythmic abilities in children with HL (Vongpaisal et al. 2016) and the absence of a thorough assessment of rhythmic skills, the aim of the present study was to gain a better understanding of rhythmic skills and their relation to speech perception abilities.

One way to assess rhythmic abilities is to use sensorimotor synchronization tasks. In these tasks, participants move (e.g., by finger tapping) to the beat of auditory rhythms of varying temporal and spectral complexity (e.g., metronome, music). These tasks require participants to analyze the complex temporal structure of the auditory signal, extract periodicities in the signal (e.g., at the beat level), organize these periodicities according to a hierarchical structure with different levels of prominence (the metrical levels). The construction of this hierarchical rhythmic representation is instrumental for generating temporal predictions. This allows listeners anticipating when the next beat will occur and thereby aligning their motor response to it (e.g., Repp 2005; Sowiński \& Dalla Bella 2013). Notably, similar temporal skills could be relevant in structuring speech sounds into a temporal hierarchical organization during speech perception (Giraud \& Poeppel 2012). Although it is agreed that speech does not present a clear periodicity and stress patterns are generally more variable than in music (Jadoul et al. 2016), both speech and music share different levels of hierarchically organized accents or prominences giving rise to stress patterns (Di Cristo 2002; Jun \& Fougeron 2002). Listeners have to identify more or less prominent speech units (Lehiste 1977; Fowler 1979) and predict their next moment of occurrence to adequately plan their own production (Garrod \& Pickering 2015). Hence rhythmic skills may be informative about speech and language capacities because shared mechanisms may support the extraction of hierarchical structures in the timing domain (Fitch \& Martins 2014; Haegens \& Zion Golumbic 2018).

While some studies have investigated rhythmic perception in children with CI in music (Hopyan et al. 2012; Innes-Brown et al. 2013; Roy et al. 2014; Good et al. 2017; Polonenko et al. 2017), few have specifically addressed rhythm production abilities, to our knowledge. We tested rhythm production in children with HL using a synchronization finger tapping task. Participants were asked to tap with one hand to the beat of different auditory rhythms consisting of simple sounds sequences or music and to reproduce complex rhythmic patterns (i.e., more complex durational patterns).

Previous studies assessing sensorimotor synchronization abilities in adults and children with HL showed normal or close to normal behavior with acoustically and structurally simple stimuli (Phillips-Silver et al. 2015; Vongpaisal et al. 2016). Thus, we hypothesized that children with HL would perform similarly to NH children in a sensorimotor synchronization task using a simple stimulus like a metronome. By contrast, we expected poorer performances in HL compared to $\mathrm{NH}$ children when using a multi-instrumental musical stimulus that requires efficient spectral processing to separate the sources and extract the beat (Pressnitzer et al. 2011). Finally, we predicted that children with HL would have lower abilities to reproduce complex rhythmic sequences compared to $\mathrm{NH}$ children. Considering that working memory capacities are important in perceiving complex rhythmic sequences (Tierney \& Kraus 2015) and that children with HL suffer from verbal working memory deficits (Nittrouer et al. 2013; AuBuchon et al. 2015), we assessed potential deficits in rhythmic production abilities by controlling for differences in auditory working memory.

Our second aim was to investigate a possible relation between temporal rhythmic production deficits and speech perception. Since both speech and music perception require an accurate processing of hierarchical stress patterns (Cason \& Schön 2012; Schön \& Tillmann 2015), and rhythmic training seems to improve stress perception (Hausen et al. 2013; Cason et al. 2015; Torppa et al. 2018; Torppa \& Huotilainen 2019), we predicted that, for children with HL, performance in speech perception would correlate with performance on imitation of complex rhythms. Indeed, this latter task requires constructing an accurate 
hierarchical stress pattern representation. The performance of children with HL was compared to that of age-matched controls and interpreted relative to auditory working memory and sentence repetition abilities, as well as CI device-related variables (onset of use, type of device, and duration of use).

\section{MATERIALS AND METHODS}

\section{Participants}

Thirty-two French-speaking children aged from 5 to 10 years $($ mean $=88.2$ months, $\mathrm{SD}=19.8$ months) with congenital, prelingual, or perilingual severe (5) to profound (26) HL group, without any known additional disorders were recruited from the pediatric implantation center of Lyon (Edouard Herriot Hospital, France) and from 4 independent speech therapy centers in Marseille (France). These children wore bilateral hearing aids (4) or one (8) or two CIs (20), and/or a contralateral hearing aid (see Supplemental Digital Content 1 for a Table with more details http://links.lww.com/EANDH/A691); were included in mainstream schools; and benefited from two speech therapy sessions per week on average. Following the recommendation of the French health authority, each session lasted $45 \mathrm{~min}$ on average and consisted of auditory, speech and language stimulation adapted to each child's individual level. Four of them had previously followed musical initiation activities. Seventeen were right-handed. Twenty-four children with NH group were recruited from two elementary schools: one in Lyon and one in Marseille (France). These children were all French native speakers without any known visual, speech, cognitive, or hearing disorder and were paired in age with children with HL (mean $=90$ months, SD = 21.2 months; no age difference between NH and HL children: $t(54)=-0.33, p=0.74)$. Seven of them had previously followed musical initiation activities, 3 had taken instrumental music classes and 18 were right-handed. This study was approved by the Ethics Committee Sud Méditerranée I (nID RCB: 2015-A01490-49).

\section{Procedure}

All children were assessed individually with three synchronization tests and a working memory test. A speech perception test was also administered to children with HL. Children were seated in front of the experimenter in a quiet room. In the synchronization tasks, they were asked to tap with the index of their dominant hand on a digital tablet (I.T. Works $10.1,25.65 \mathrm{~cm}$ ) or onto a cardboard box containing a ZOOM H4n digital recorder. All sounds were delivered by loudspeakers (Creative Inspire T1) placed at $1 \mathrm{~m}$ from the child. Sound volume was manually adjusted for every child to a comfortable loudness level. A training phase with the experimenter preceded each task. In this phase, we could ensure that each child could hear the stimuli. The experimental session lasted approximately $30 \mathrm{~min}$.

\section{Tasks Description}

First, children were asked to tap regularly at their preferred pace, without an auditory context (spontaneous tapping). Then, they were asked to synchronize their finger tapping to the beat of various rhythmic sequences of varying complexity, namely a metronome, music excerpts, and rhythmic sequences. Synchronization to a metronome (paced tapping) was tested using the BAASTA battery (Dalla Bella et al. 2017b) implemented on a tablet device (Bégel et al. 2017). Children were asked to tap in synchrony with 60 isochronous piano tones (tone frequency $=1319 \mathrm{~Hz}$, pitch $=\mathrm{E} 5$, interonset interval of $600 \mathrm{~ms}$ ). Synchronization to music was tested using the stimuli from the Beat Alignment Test (Iversen \& Patel 2008). Children were asked to tap in synchrony with the beat of one jazz (Tuxedo Junction of Glenn Miller) and one pop (A Chorus Line of Boston Pops) musical excerpts (music tapping). Each excerpt lasted 17 and $14 \mathrm{sec}$, respectively, and was repeated twice (54 and 58 taps, respectively, tempo of $\sim 90$ and $120 \mathrm{bpm}$ ). Synchronization with complex rhythmic sequences was assessed using the protocol of Tierney and Kraus (2015). In this task, children listened to a complex rhythm (cf. Supplemental Digital Content 2, http://links.lww.com/EANDH/A689, for the Audio stimuli, tempo of $120 \mathrm{bpm}$ ) that was repeated eight times in a row, and they were asked to imitate the whole rhythmic pattern (and not the beat) by tapping along the rhythm (imitation of complex rhythms). Importantly, the use of one single sound with a short attack time and a short duration ensures a low spectral complexity of these stimuli.

The speech perception task consisted of repeating very short sentences. Twenty French, syntactically simple sentences (subject-verb-complement) were selected from Cason et al. (2015). Each sentence was composed of six syllables (cf. Supplemental Digital Content 3 for the Audio stimuli, http://links.lww.com/ EANDH/A690). Children listened to each sentence twice and were then asked to repeat it. Because the aim of this test was mostly to assess speech perception, the scoring realized by a speech therapist only considered segmental errors and not prosodic errors or misarticulations. One point was attributed for each correctly repeated syllable (one or more wrong phonemes in a syllable-vowel or consonant - counted as an error); the final mark was the average for the 20 sentences which was then converted into percentage of correct syllables. This test was previously piloted with $\mathrm{NH}$ children who scored at ceiling.

A working memory test was selected from the Wechsler Intelligence Scale for Children IV (indirect span). Children were asked to repeat sequences of digits of increasing length in the reverse order. The test stops when the child gives a wrong answer on more than two items of a given length in a row.

\section{Analyses of Tapping to the Beat}

Unpaced and tapping times with metronome and musical excerpts were plotted on a polar scale in which each tap is represented on a circle of $360^{\circ}$ by an angle relative to the expected beat time $\left(=0^{\circ}\right.$ on the circle). Taps in a trial are treated as unitary vectors; the resulting vector is calculated to quantify sensorimotor synchronization to the beat (for a description of the procedure, common in tapping studies, see Sowiński \& Dalla Bella 2013). The length of this vector, named synchronization consistency (from 0 to 1 ), is a measure of synchronization performance $(0=$ performance at chance; $1=$ perfect phase synchronization). Vector length was logit transformed for group statistics when necessary (Falk et al. 2015).

\section{Analysis of the Imitation of Complex Rhythmic Sequences}

For each repetition of the sequence out of the eight repetitions, the first three were considered as a learning phase; hence, only the last five repetitions were analyzed. Analyses were on 
average run on 180 taps per child. First, we extracted the temporal series of tapping from the audio recording. To this aim, we used the SciPy function find_peaks with a minimal distance of $100 \mathrm{~ms}$ and a prominence of 2 SD. We developed a new objective method to score children's performances, based on computing a correlation between children's tap timing and the rhythms to be reproduced. To this aim, we modeled three sources of noise: temporal jitters (small lags), temporal lags (large lags), and small accelerations/decelerations. To account for temporal jitters, we first convolved each tapping performance with a Gaussian filter $(\mathrm{SD}=50 \mathrm{~ms})$. Then, to account for temporal lags and small accelerations/decelerations we performed the correlations between the stimulus time series and each combination of temporally stretched performances ( 85 to $115 \%$ of the performance duration, 50 steps) and lags. The score of each performance was defined as the maximum value of these correlations (see Supplemental Digital Content 4 for the Python Script, http://links.lww.com/EANDH/A692). To ascertain the reliability of this objective measure, we compared it to the subjective scoring of three expert judges (blind to the children group membership). The independent judges scored each item performance using a 1 to 9 scale (very poor to excellent rhythmic performance). Spearman correlations between the objective measure and the judges scores were strong (correlation between the objective measure and judge $1: r=0.86$; judge 2: $r=0.77$; judge $3: r=0.79$; correlation with the mean of the three judges: $r=0.86$ ) and equivalent to interjudges correlations (correlations between judges 1 and 2: $r=0.83$, between judges 1 and $3 r=0.81$; between judges 2 and $3: r=0.77$ ), thus confirming the reliability of the objective measure.

\section{Statistical Modeling}

All statistical analysis was computed using R (R Core Team, 2018). We computed linear regressions for all variables with group (hearing status) as a predictor variable. These analyses were implemented using the $\operatorname{lm}()$ function, from the lme4 package (Bates et al. 2015). As children's musical experience could have an influence on the results, we modeled musical experience as a confounding factor (categorical) in all the linear models. Thereby, the potential influence of musical experience can be assessed and factored out. We tested all the assumptions of the models using the Global Validation of Linear Model Assumptions package. When the assumption of normality was not accepted, we computed a logit transformation on the data.

To control for a possible effect of working memory on the imitation of complex rhythms performance, we also included working memory as a continuous predictor variable. For children with HL only, performances in each task were modeled with duration, type of device, and onset of use as additional predictors in the linear model. Then, we computed model comparison on Akaike Information Criterion (AIC) which is a standard measure to arbitrate between complexity and explanatory power of the models. We report the model with the lowest AIC.

\section{RESULTS}

Variability in spontaneous tapping did not differ between groups $(\beta=0.01, \mathrm{SE}=0.06, t=0.23, p>0.05)$. Children with HL tapped less consistently with the metronome (paced tapping) and with the different musical excerpts (music tapping) compared to $\mathrm{NH}$ children $(\beta=-1.32, \mathrm{SE}=0.47, t=-2.77$, $p=0.007$, Cohen's $d=0.55 ; \beta=-0.23, \mathrm{SE}=0.05, t=-3.92, p$ $<0.001$, Cohen's $d=1.12$; see Fig. 1, panels A and B, respectively). Rayleigh test (an estimate of the uniformity of the taps distribution) also showed that more children with HL tapped randomly with the metronome compared to children with $\mathrm{NH}\left(\chi^{2}=6.74, p=0.009\right)$. They also performed more poorly than children with $\mathrm{NH}$ in the imitation of complex rhythms ( $\beta=-0.099, \mathrm{SE}=0.02, t=-4.65, p<0.001$, Cohen's $d=1.38)$ (see Fig. 1, panel C). This difference holds true even when controlling for working memory $(\beta=0.07, \mathrm{SE}=0.01, t=3.53, p<$ 0.001 ; correlation between indirect span and imitation of complex rhythms performances $r=0.53, p<0.01$ ) which showed a poorer performance in children with $\mathrm{HL}(\beta=-1.36, \mathrm{SE}=0.53$, $t=-2.56, p=0.01$, Cohen's $d=0.67$, see Fig. 1 , panel D).

Musical experience did not have an effect on any of the dependent variables previously reported (all $p \mathrm{~s}>0.4$ ); this was not surprising considering the short duration and weak intensity of the overall musical experience (musical initiation activities).

To evaluate links between speech and sensorimotor synchronization abilities in children with HL, we then computed linear regressions including sentence repetition scores, indirect span and imitation of complex rhythms, in children with HL only. Results showed, as expected, a relation between sentence repetition and indirect $\operatorname{span}(\beta=0.46, \mathrm{SE}=0.17, t=2.59, p=0.01)$. Interestingly, we also found that the performance in the imitation of complex rhythms task explained sentence repetition scores $(\beta=0.39, \mathrm{SE}=0.17, t=2.214, p=0.03$, see Fig. 2$)$.

The regression analysis showed that duration and onset of device use well predicted performance in tapping consistency (metronome: $\mathrm{F}=4.45, p=0.04$, $\mathrm{AIC}=19.66$; music: $\mathrm{F}=3.47, p=0.07$, AIC $=-19.27)$, imitation of complex rhythms $(\mathrm{F}=11.05, p=0.002, \mathrm{AIC}=-89.50)$, and indirect span $(\mathrm{F}=7.28, p=0.01, \mathrm{AIC}=106.24)$. Sentence repetition performance was only predicted by duration of device use $(\mathrm{F}=8.86$, $p=0.006, \mathrm{AIC}=277.83)$. The device type did not predict performance in any of the administered tasks.

\section{DISCUSSION}

In this study, we investigated rhythmic production abilities in congenitally deaf children with HL. We assessed sensorimotor synchronization to the beat of nonverbal stimuli of different acoustic and rhythmic complexity. We have also assessed working memory in both populations and sentence repetition in children with HL. Children with HL revealed poorer motor synchronization to the beat as compared to their NH peers. To our knowledge, this is the first time that this deficit is clearly isolated in children with HL. We will first discuss the basis of this deficit in relation to potential difficulties encountered by children with HL in realizing hierarchical temporal processing of auditory information. Second, we will discuss the links between the rhythmic competences of children with HL and speech competences.

\section{Congenitally Deaf Children Have Poor Sensorimotor Synchronization Abilities}

Our results show that children with HL perform worse than children with $\mathrm{NH}$ when tapping to a metronome, music, and complex rhythmical sequences. These results suggest that HL may have altered the abilities involved in coupling movement to the beat of an auditory stimulus (Phillips-Silver et al. 2015). 
A

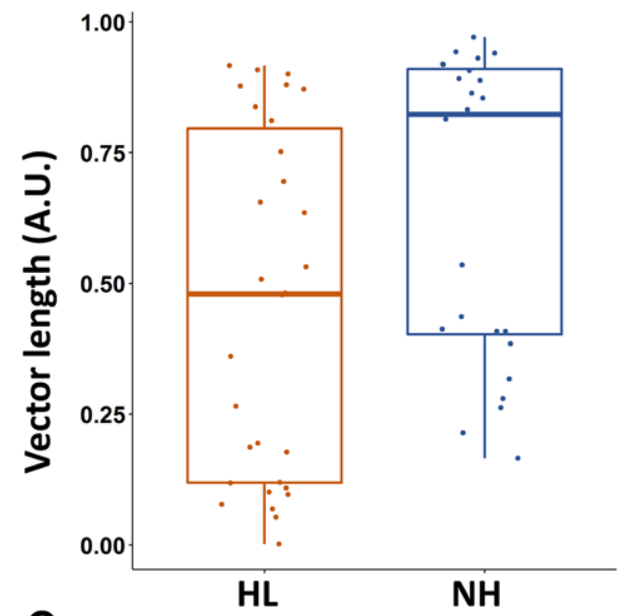

c

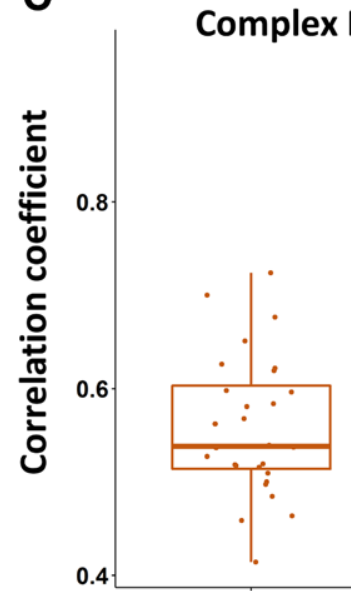

HL
B
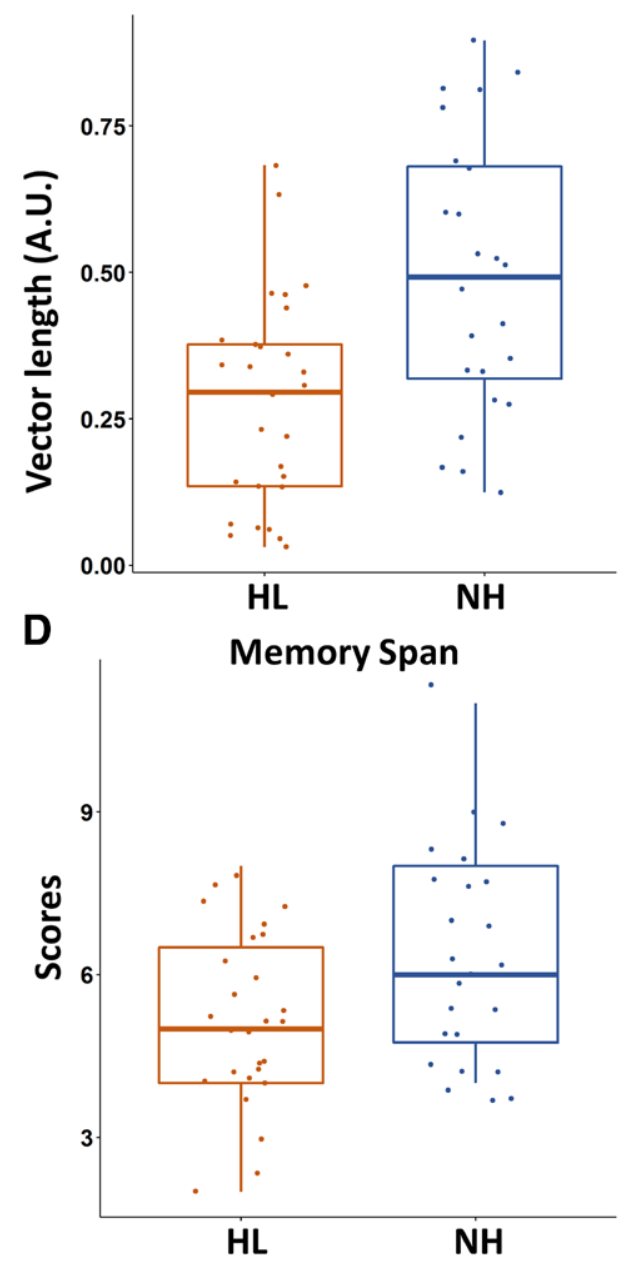

Fig. 1. Illustration of the results in the sensorimotor synchronization tasks and the memory task. A and B, Synchronization consistency measured for HL and $\mathrm{NH}$ children's tapping along with a metronome and musical excerpts. Higher values represent better performances (range 0 to 1 ). $\mathrm{C}$, $\mathrm{HL}$ and $\mathrm{NH}$ children's synchronization performances on the imitation of complex rhythms task represented by the correlation coefficient between the stimuli and the children taps. $\mathrm{D}, \mathrm{HL}$ and $\mathrm{NH}$ scores in the working memory task. HL indicates hearing loss; $\mathrm{NH}$, normal-hearing. Errors bars represent the standard error of the mean.

Importantly, these deficits cannot be ascribed to a general motor timing deficit, as children showed unimpaired performance when tapping without a stimulus. One possibility is that poor sensorimotor synchronization reflects a general rhythmic perception deficit in children with HL (see Stabej et al. 2012). However, not all studies support this hypothesis. In a preliminary study, Innes-Brown et al. (2013) tested the musical perceptual abilities of NH children $(n=8)$, implanted children $(n=6)$, and children with hearing aids $(n=5)$. Children with HL scored lower than $\mathrm{NH}$ children in pitch discrimination and timbre recognition tasks but showed a similar performance in rhythmic discrimination. Interestingly, while the degree of HL was a good predictor of performance on the pitch and timbre tests, it did not predict rhythmic performance. Similarly to children with HL, postlingually deaf adults can better appreciate the rhythmic than the melodic structure of musical stimuli (McDermott 2004; Looi et al. 2008). Adults with CI perform similarly to $\mathrm{NH}$ adults in the discrimination or identification of rhythmic patterns, as well as tempo discrimination, but are less skilled in tone or melody discrimination (Collins et al. 1994; Gfeller et al. 1997; Kong et al. 2004). Unless in a complex auditory scene with many similar instruments, rhythm perception does not necessarily require a fine spectral analysis. Considering the technical limitations of the CI in terms of spectral processing and the rather good performance in terms of temporal processing of the auditory signal (Limb \& Roy 2014), the different performance of adults with CI in melodic and rhythmic tasks might be interpreted in terms of the acoustic nature of the stimuli to be processed. The poor spectral resolution of CIs could also account for some of our results with children with HL. Their greater difficulty to synchronize to the beat of musical excerpts compared to the performance with the metronome is in line with results found in adults (Sowiński \& Dalla Bella 2013; Dalla Bella et al. 2017a) and NH children populations (Puyjarinet et al. 2017). Specifically for children with HL, this difficulty could be partly due to the greater demands in terms of auditory scene analysis which heavily involves spectral analysis to elaborate and select objects in a complex auditory environment (Oxenham 2008; ShinnCunningham et al. 2017).

However, children with HL also performed more poorly than the control group when synchronizing to a spectrally very simple stimulus (auditory metronome) and when tapping to complex rhythmic tone sequences that did not require complex spectral processing. Thus, a poor spectral representation cannot 


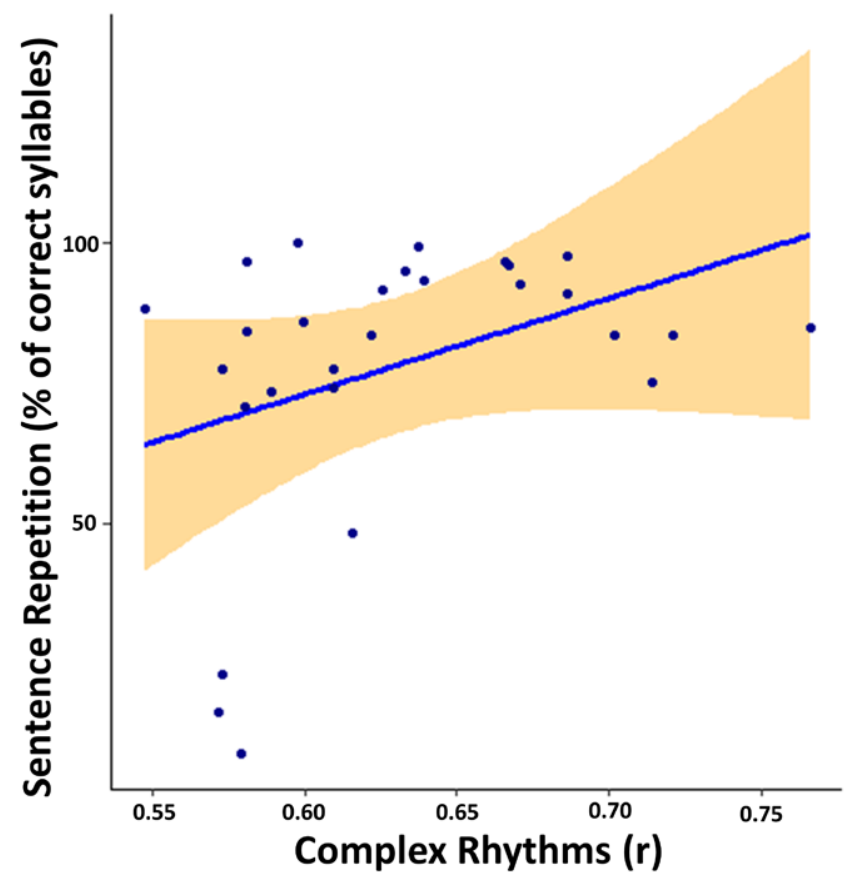

Fig. 2. Relation between sentence repetition scores in percentage of correct syllables and performance in the imitation of complex rhythms task measured by the coefficient of correlation between children's taps timing and the rhythms be reproduced. Shaded area represents confidence intervals (0.99).

fully account for our results and a temporal processing deficit seems to be present in children with HL, similarly to findings with deaf adults. Phillips-Silver et al. (2015) asked postlingually deaf adults with $\mathrm{CI}$ and adults with $\mathrm{NH}$ to synchronize to stimuli with different spectral complexity: a song, a version of the song with reduced spectral complexity (played on the piano) and a version played on percussions containing only the rhythmic structure. Regardless of spectral complexity, adults with CI displayed a poorer tapping performance than adults with NH in all the tasks except when tapping to a visual metronome (Phillips-Silver et al. 2015).

When evaluating a potential temporal processing deficit in children with HL, it is important to carefully examine the possible role of working memory. Overall, we replicated previous studies showing a working memory deficit in children with HL children (Pisoni \& Cleary 2003; Beer et al. 2011). Tapping to complex rhythms may have been particularly affected by these memory restrictions. However, it is important to recall that in this task, children tap along several repetitions of the rhythmic sequence. Reproducing several times the same rhythm and tapping along with it are both supposed to reduce working memory load. Moreover, we showed that, even when modeling working memory variability, the performance in the complex rhythms task remained significantly worse in children with HL compared to children with NH. Thus, memory skills of children with HL cannot entirely account for their deficit in this task.

In a NH population, sensorimotor synchronization requires temporal predictions affording fine coupling between the auditory and motor systems (Chen et al. 2008; Patel \& Iversen 2014). The poor tapping abilities of children with HL, present with simple stimuli but exacerbated with stimuli of increasing temporal complexity, might stem from difficulties in temporal anticipation of the beat and/or motor adaptation. As suggested by Gfeller et al. (1997) for postlingually deaf adults with CI, rhythmic difficulties may be accompanied by a higher-level temporal deficit related to the structuring of acoustic events in a hierarchical manner.

\section{Sensorimotor Synchronization Deficits and Speech Abilities}

Potential difficulties for congenitally children with HL in perceiving a temporal hierarchical organization of sounds may have affected their ability to perceive speech. This idea is supported by our finding that children's performance in the imitation of complex rhythms task correlates with performance in sentence repetition.

The ability to organize sequential auditory events according to a hierarchical structure would indeed be of utmost importance in auditory perception of speech. For example, speech sounds have to be organized as patterns of more or less prominent perceptual units (e.g., accent patterns featuring alternation of strong and weak syllables, see Hirst \& Di Cristo 1998). Interestingly, priming the temporal structure of speech using a clear musical beat and meter facilitates speech perception possibly via increased neural coupling to the phonological structure (Cason et al 2015; Falk et al 2017). The field of metrical phonology shows that building a correct hierarchy of syllabic prominence levels is essential to identify utterance boundaries and communicative intent of these utterances (e.g., Post 2012). To put it differently, structuring auditory information in a temporal hierarchical structure may be needed to identify the different levels of the entire hierarchy of speech.

Nonetheless, the present results only show a medium correlation between rhythmic and speech abilities). Thus, while consistent with a literature showing a link between rhythmic and speech abilities in normal (Tierney \& Kraus 2013b; Ozernov-Palchik et al. 2018) as well as in developmental disorders (Muneaux et al. 2004; Corriveau \& Goswami 2009; Wieland et al. 2015), our results need to be taken with care and stronger claims on this link await further research possibly focusing on the relation between rhythm and speech production abilities.

\section{CONCLUSION}

In sum, it is possible that despite early cochlear implantation or hearing aid use, hearing impairment may affect the quality of temporal processing of acoustic stimuli in congenitally deaf children. The sensorimotor synchronization deficit of children with HL found in this study, possibly linked to an underlying deficit in temporal motor anticipation/adaptation, suggests that future studies should also examine how HL in early childhood affects the audio-motor network in the brain of children with HL and its contribution to anticipating rhythmic events and adapting a motor response to these events (e.g., van der Steen \& Keller 2013).

Finally, the link between speech capacities and sensorimotor synchronization in children with HL should be examined in more detail. As recommended by studies conducted in other clinical populations with language and speech deficits (Przybylski et al. 2013; Flaugnacco et al. 2015), the effect of musical training, and more specifically rhythmic training, could be examined in relation to the ability to perceive and produce speech in children with HL. Interestingly, the efficiency of this type of interventions may also depend upon the level of 
impairment of sensorimotor synchronization abilities, as suggested for other populations (e.g., Dalla Bella et al. 2018).

\section{ACKNOWLEDGMENTS}

We thank Valentin Bégel for his contribution in BAASTA tapping analysis.

This research was supported by grants of the Fondation Agir pour l'Audition (APA RD-2016-9), the ANR-16-CE28-0012 RALP, ANR-11-LABX-0036 (BLRI), ANR-16-CONV-0002 (ILCB), and the Excellence Initiative of Aix-Marseille University (A*MIDEX).

C.H., D.S., and S.D.B. designed the study; A.Z. and C.H. acquired data; C.H. and S.D.B. analyzed the data; C.H., D.S., S.D.B., S.F., E.T, and S.R. wrote the manuscript.

The authors have no conflicts of interest to disclose.

Address for correspondence: Céline Hidalgo, Institut de Neurosciences des Systèmes, UMR 1106, Aix-Marseille University, Faculté de Médecine de la Timone, 27 Boulevard Jean Moulin, 13005 Marseille, France. E-mail: celine.hidalgo@univ-amu.fr

Received June 14, 2019; accepted June 19, 2020.

\section{REFERENCES}

AuBuchon, A. M., Pisoni, D. B., Kronenberger, W. G. (2015). Short-term and working memory impairments in early-implanted, long-term cochlear implant users are independent of audibility and speech production. Ear Hear, 36, 733-737.

Baldassari, C. M., Schmidt, C., Schubert, C. M., et al. (2009). Receptive language outcomes in children after cochlear implantation. Otolaryngol Head Neck Surg, 140, 114-119.

Bandurski, M., \& Galkowski, T. (2004). The development of analogical reasoning in deaf children and their parents' communication mode. J Deaf Stud Deaf Educ, 9, 153-175.

Bates, D., Mächler, M., Bolker, B., Walker, S. (2015). Fitting linear mixedeffects models using $\{1 \mathrm{me} 4\}$. J Stat Softw, 67, 1-48.

Bedoin, N., Brisseau, L., Molinier, P., et al. (2016). Temporally regular musical primes facilitate subsequent syntax processing in children with specific language impairment. Front Neurosci, 10, 245.

Beer, J., Kronenberger, W. G., Pisoni, D. B. (2011). Executive function in everyday life: Implications for young cochlear implant users. Cochlear Implants Int, 12(Suppl 1), S89-S91.

Bégel, V., Benoit, C. E., Correa, A., et al. (2017). "Lost in time" but still moving to the beat. Neuropsychologia, 94, 129-138.

Blamey, P. J., Sarant, J. Z., Paatsch, L. E., et al. (2001). Relationships among speech perception, production, language, hearing loss, and age in children with impaired hearing. $J$ Speech Lang Hear Res, 44, 264-285.

Boons, T., De Raeve, L., Langereis, M., et al. (2013a). Expressive vocabulary, morphology, syntax and narrative skills in profoundly deaf children after early cochlear implantation. Res Dev Disabil, 34, 2008-2022.

Boons, T., De Raeve, L., Langereis, M., et al. (2013b). Narrative spoken language skills in severely hearing impaired school-aged children with cochlear implants. Res Dev Disabil, 34, 3833-3846.

Bourguignon, M., De Tiège, X., de Beeck, M. O., et al. (2013). The pace of prosodic phrasing couples the listener's cortex to the reader's voice. Hum Brain Mapp, 34, 314-326.

Caselli, M.C., Rinaldi, P., Varuzza, C., et al. (2012). Cochlear implant in the second year of life: Lexical and grammatical outcomes. $J$ Speech Lang Hear Res, 55, 382-394.

Cason, N., \& Schön, D. (2012). Rhythmic priming enhances the phonological processing of speech. Neuropsychologia, 50, 2652-2658.

Cason, N., Hidalgo, C., Isoard, F., et al. (2015). Rhythmic priming enhances speech production abilities: Evidence from prelingually deaf children. Neuropsychology, 29, 102-107.

Chen, J. L., Penhune, V. B., Zatorre, R. J. (2008). Moving on time: Brain network for auditory-motor synchronization is modulated by rhythm complexity and musical training. J Cogn Neurosci, 20, 226-239.

Chobert, J., Marie, C., François, C., et al. (2011). Enhanced passive and active processing of syllables in musician children. J Cogn Neurosci, 23, 3874-3887.
Cochen De Cock, V., Dotov, D.G., Ihalainen, P., et al. (2018). Rhythmic abilities and musical training in Parkinson's disease: Do they help? Park Dis, 4,8 .

Collins, L. M., Wakefield, G. H., Feinman, G. R. (1994). Temporal pattern discrimination and speech recognition under electrical stimulation. $J$ Acoust Soc Am, $96(5$ Pt 1), 2731-2737.

Conway, C. M., Pisoni, D. B., Anaya, E. M., et al. (2011). Implicit sequence learning in deaf children with cochlear implants. Dev Sci, 14, 69-82.

Corriveau, K. H., \& Goswami, U. (2009). Rhythmic motor entrainment in children with speech and language impairments: Tapping to the beat. Cortex, 45, 119-130

Dalla Bella, S., Benoit, C. E., Farrugia, N., et al. A. (2017a). Gait improvement via rhythmic stimulation in Parkinson's disease is linked to rhythmic skills. Sci Rep, 7, 42005.

Dalla Bella, S., Dotov, D., Bardy, B., de Cock, V. C. (2018). Individualization of music-based rhythmic auditory cueing in Parkinson's disease. Ann N Y Acad Sci, 1423, 308-317.

Dalla Bella, S., Farrugia, N., Benoit, C. E., et al. (2017b). BAASTA: Battery for the assessment of auditory sensorimotor and timing abilities. Behav Res Methods, 49, 1128-1145.

Di Cristo, A. (2002). De la métrique et du rythme de la parole ordinaire: L'exemple du français. In E. Bordas (Ed.), Le Rythme de la Prose (pp. 25-44). Presses Universitaires de Franche-Comté.

Ding, N., \& Simon, J. Z. (2014). Cortical entrainment to continuous speech: Functional roles and interpretations. Front Hum Neurosci, 8, 311.

Ding, N., Melloni, L., Zhang, H., et al. (2016). Cortical tracking of hierarchical linguistic structures in connected speech. Nat Neurosci, 19, $158-164$.

Doelling, K. B., Arnal, L. H., Ghitza, O., Poeppel, D. (2014). Acoustic landmarks drive delta-theta oscillations to enable speech comprehension by facilitating perceptual parsing. Neuroimage, 85(Pt 2), 761-768.

Falk, S., Lanzilotti, C., Schön, D. (2017). Tuning neural phase entrainment to speech. J Cogn Neurosci, 29, 1378-1389.

Falk, S., Müller, T., Dalla Bella, S. (2015). Non-verbal sensorimotor timing deficits in children and adolescents who stutter. Front Psychol, 6, 847.

Fitch, W. T., \& Martins, M. D. (2014). Hierarchical processing in music, language, and action: Lashley revisited. Ann NY Acad Sci, 1316, 87-104.

Flaugnacco, E., Lopez, L., Terribili, C., et al. (2015). Music training increases phonological awareness and reading skills in developmental dyslexia: A randomized control trial. PLoS One, 10, e0138715.

Flaugnacco, E., Lopez, L., Terribili, C., et al. (2014). Rhythm perception and production predict reading abilities in developmental dyslexia. Front Hum Neurosci, 8, 392.

Fowler, C.A. (1979). "Perceptual centers" in speech production and perception. Percept Psychophys, 25, 375-388.

Garrod, S., \& Pickering, M. J. (2015). The use of content and timing to predict turn transitions. Front Psychol, 6, 751.

Geers, A. E., Strube, M. J., Tobey, E. A., et al. (2011). Epilogue: Factors contributing to long-term outcomes of cochlear implantation in early childhood. Ear Hear, 32(1 Suppl), 84S-92S.

Gfeller, K., Woodworth, G., Robin, D. A., et al. (1997). Perception of rhythmic and sequential pitch patterns by normally hearing adults and adult cochlear implant users. Ear Hear, 18, 252-260.

Giraud, A. L., \& Poeppel, D. (2012). Cortical oscillations and speech processing: Emerging computational principles and operations. Nat Neurosci, $15,511-517$.

Good, A., Gordon, K. A., Papsin, B. C., et al. (2017). Benefits of music training for perception of emotional speech prosody in deaf children with cochlear implants. Ear Hear, 38, 455-464.

Grandon, B., Martinez, M. J., Samson, A., Vilain, A. (2020). Long-term effects of cochlear implantation on the intelligibility of speech in Frenchspeaking children. $J$ Child Lang, 47, 881-892.

Habib, M. G., Waltzman, S. B., Tajudeen, B., Svirsky, M. A. (2010). Speech production intelligibility of early implanted pediatric cochlear implant users. Int J Pediatr Otorhinolaryngol, 74, 855-859.

Haegens, S., \& Zion Golumbic, E. (2018). Rhythmic facilitation of sensory processing: A critical review. Neurosci Biobehav Rev, 86, 150-165.

Hausen, M., Torppa, R., Salmela, V. R., et al. (2013). Music and speech prosody: A common rhythm. Front Psychol, 4, 566.

Hayes, H., Geers, A. E., Treiman, R. (2009). Receptive vocabulary development in deaf children with cochlear implants: Achievement in an intensive auditory-oral educational setting. Ear Hear, 30, 128-135.

Hidalgo, C., Falk, S., Schön, D. (2017). Speak on time! Effects of a musical rhythmic training on children with hearing loss. Hear Res, 351, 11-18. 
Hidalgo, C., Pesnot-Lerousseau, J., Marquis, P., et al. (2019). Rhythmic training improves temporal anticipation and adaptation abilities in children with hearing loss during verbal interaction. J Speech Lang Hear Res, 62, 3234-3247.

Hirst, D.J., \& Di Cristo, A. (1998). A survey of intonation systems. In D. J. Hirst \& A. Di Cristo (Eds.), Intonation Systems: A Survey of Twenty Languages (pp. 1-44). Cambridge University Press.

Holt, C. M., Demuth, K., Yuen, I. (2016). The use of prosodic cues in sentence processing by prelingually deaf users of cochlear implants. Ear Hear, 37, e256-e262.

Holt, C. M., Yuen, I., Demuth, K. (2017). Discourse strategies and the production of prosody by prelingually deaf adolescent cochlear implant users. Ear Hear, 38, e101-e108.

Hopyan, T., Peretz, I., Chan, L. P., et al. (2012). Children using cochlear implants capitalize on acoustical hearing for music perception. Front Psychol, 3, 425.

Houston, D. M., \& Bergeson, T. R. (2014). Hearing versus listening: Attention to speech and its role in language acquisition in deaf infants with cochlear implants. Lingua, 139, 10-25.

Houston, D. M., Pisoni, D. B., Kirk, K. I., et al. (2003). Speech perception skills of deaf infants following cochlear implantation: A first report. Int $J$ Pediatr Otorhinolaryngol, 67, 479-495.

Innes-Brown, H., Marozeau, J. P., Storey, C. M., Blamey, P. J. (2013). Tone, rhythm, and timbre perception in school-age children using cochlear implants and hearing aids. J Am Acad Audiol, 24, 789-806.

Iversen J. R., \& Patel, A. D. (2008). The Beat Alignment Test (BAT): Surveying beat processing abilities in the general population. In Proceedings of the $10^{\text {th }}$ Internation Conference on Music Perception and Cognition (ICMPC 10), 465-468.

Jadoul, Y., Ravignani, A., Thompson, B., et al. (2016). Seeking temporal predictability in speech: Comparing statistical approaches on 18 world languages. Front Hum Neurosci, 10, 586.

Jiam, N. T., Caldwell, M., Deroche, M. L., et al. (2017). Voice emotion perception and production in cochlear implant users. Hear Res, 352, 30-39.

Johnson, C., \& Goswami, U. (2010). Phonological awareness, vocabulary, and reading in deaf children with cochlear implants. J Speech Lang Hear Res, 53, 237-261.

Jun, S.-A., \& Fougeron, C. (2002). Realizations of accentual phrase in French intonation. Probus, 14, 147-172.

Kong, Y. Y., Cruz, R., Jones, J. A., Zeng, F. G. (2004). Music perception with temporal cues in acoustic and electric hearing. Ear Hear, 25, 173-185.

Kösem, A., \& van Wassenhove, V. (2017). Distinct contributions of low- and high-frequency neural oscillations to speech comprehension. Lang Cogn Neurosci, 32, 536-544.

Kotz, S. A., \& Schwartze, M. (2010). Cortical speech processing unplugged: A timely subcortico-cortical framework. Trends Cogn Sci, 14, 392-399.

Lehiste, I. (1977). Isochrony reconsidered. J Phon, 5, 253-263.

Limb, C. J., \& Roy, A. T. (2014). Technological, biological, and acoustical constraints to music perception in cochlear implant users. Hear Res, 308, $13-26$.

Looi, V., McDermott, H., McKay, C., Hickson, L. (2008). Music perception of cochlear implant users compared with that of hearing aid users. Ear Hear, 29, 421-434.

Lund, E. (2016). Vocabulary knowledge of children with cochlear implants: A meta-analysis. J Deaf Stud Deaf Educ, 21, 107-121.

Luo, H., \& Poeppel, D. (2007). Phase patterns of neuronal responses reliably discriminate speech in human auditory cortex. Neuron, 54, 1001-1010.

McDermott, H. J. (2004). Music perception with cochlear implants: A review. Trends Amplif, 8, 49-82.

Muneaux, M., Ziegler, J. C., Truc, C., et al. (2004). Deficits in beat perception and dyslexia: Evidence from French. Neuroreport, 15, 1255-1259.

Nittrouer, S., Caldwell-Tarr, A., Lowenstein, J. H. (2013). Working memory in children with cochlear implants: Problems are in storage, not processing. Int J Pediatr Otorhinolaryngol, 77, 1886-1898.

Oxenham, A. J. (2008). Pitch perception and auditory stream segregation: Implications for hearing loss and cochlear implants. Trends Amplif, 12, 316-331.

Ozernov-Palchik, O., Wolf, M., Patel, A. D. (2018). Relationships between early literacy and nonlinguistic rhythmic processes in kindergarteners. $J$ Exp Child Psychol, 167, 354-368.

Patel, A. D., \& Iversen, J. R. (2014). The evolutionary neuroscience of musical beat perception: The Action Simulation for Auditory Prediction (ASAP) hypothesis. Front Syst Neurosci, 8, 57.
Peelle, J. E., \& Davis, M. H. (2012). Neural oscillations carry speech rhythm through to comprehension. Front Psychol, 3, 320.

Peng, S. C., Spencer, L. J., Tomblin, J. B. (2004). Speech intelligibility of pediatric cochlear implant recipients with 7 years of device experience. $J$ Speech Lang Hear Res, 47, 1227-1236.

Phillips-Silver, J., Toiviainen, P., Gosselin, N., et al. (2015). Cochlear implant users move in time to the beat of drum music. Hear Res, 321, 25-34.

Pisoni, D. B., \& Cleary, M. (2003). Measures of working memory span and verbal rehearsal speed in deaf children after cochlear implantation. Ear Hear, 24(1 Suppl), 106S-120S.

Polonenko, M. J., Giannantonio, S., Papsin, B. C., et al. (2017). Music perception improves in children with bilateral cochlear implants or bimodal devices. J Acoust Soc Am, 141, 4494.

Post, B. (2012). Metrical structure and the prosodic hierarchy. In P. Rebusschat, M. Rohrmeier, J. Hawkins \& I. Cross (Eds.), Language and Music as Cognitive Systems (p. 3). University Press.

Pressnitzer, D., Suied, C., Shamma, S. A. (2011). Auditory scene analysis: The sweet music of ambiguity. Front Hum Neurosci, 5, 158.

Przybylski, L., Bedoin, N., Krifi-Papoz, S., et al. (2013). Rhythmic auditory stimulation influences syntactic processing in children with developmental language disorders. Neuropsychology, 27, 121-131.

Puyjarinet, F., Bégel, V., Lopez, R., et al. (2017). Children and adults with attention-deficit/hyperactivity disorder cannot move to the beat. Sci Rep, 7,11550

R Core Team. (2013). R: A language and environment for statistical computing.

Rammsayer, T., \& Altenmüller, E. (2006). Temporal information processing in musicians and nonmusicians. Music Percept, 24, 37-48.

Repp, B. H. (2005). Sensorimotor synchronization: A review of the tapping literature. Psychon Bull Rev, 12, 969-992.

Roy, A. T., Scattergood-Keepper, L., Carver, C., et al. (2014). Evaluation of a test battery to assess perception of music in children with cochlear implants. JAMA Otolaryngol Head Neck Surg, 140, 540-547.

Sares, A. G., Foster, N. E. V., Allen, K., Hyde, K. L. (2018). Pitch and time processing in speech and tones: The effects of musical training and attention. J Speech Lang Hear Res, 61, 496-509.

Schön, D., \& Tillmann, B. (2015). Short- and long-term rhythmic interventions: Perspectives for language rehabilitation. Ann N Y Acad Sci, 1337, $32-39$.

Segal, O., \& Kishon-Rabin, L. (2011). Listening preference for childdirected speech versus nonspeech stimuli in normal-hearing and hearingimpaired infants after cochlear implantation. Ear Hear, 32, 358-372.

Shinn-Cunningham, B., Best, V., Lee, A. K. C. (2017). Auditory object formation and selection. In J. C. Middlebrooks, J. Z. Simon, A. N. Popper, R. R. Fay (Eds.), The Auditory System at the Cocktail Party (pp. 7-40). Springer International Publishing.

Sowiński, J., \& Dalla Bella, S. (2013). Poor synchronization to the beat may result from deficient auditory-motor mapping. Neuropsychologia, $51,1952-1963$.

Stabej, K. K., Smid, L., Gros, A., et al. (2012). The music perception abilities of prelingually deaf children with cochlear implants. Int $J$ Pediatr Otorhinolaryngol, 76, 1392-1400.

Thomson, J. M., \& Goswami, U. (2008). Rhythmic processing in children with developmental dyslexia: Auditory and motor rhythms link to reading and spelling. J Physiol Paris, 102, 120-129.

Tierney, A., \& Kraus, N. (2013a). Music training for the development of reading skills. Prog Brain Res, 207, 209-241.

Tierney, A., \& Kraus, N. (2013b). The ability to move to a beat is linked to the consistency of neural responses to sound. J Neurosci, 33, 14981-14988.

Tierney, A., \& Kraus, N. (2014). Auditory-motor entrainment and phonological skills: Precise auditory timing hypothesis (PATH). Front Hum Neurosci, 8, 949.

Tierney, A., \& Kraus, N. (2015). Evidence for multiple rhythmic skills. PLoS One, 10, 0136645.

Torppa, R., \& Huotilainen, M. (2019). Why and how music can be used to rehabilitate and develop speech and language skills in hearing-impaired children. Hear Res, 380, 108-122.

Torppa, R., Faulkner, A., Huotilainen, M., et al. (2014). The perception of prosody and associated auditory cues in early-implanted children: The role of auditory working memory and musical activities. Int $J$ Audiol, 53, 182-191.

Torppa, R., Faulkner, A., Kujala, T., et al. (2018). Developmental links between speech perception in noise, singing, and cortical processing 
of music in children with cochlear implants. Music Percept, 36, $156-174$

Truy, E., Lina-Granade, G., Jonas, A. M., et al. (1998). Comprehension of language in congenitally deaf children with and without cochlear implants. Int J Pediatr Otorhinolaryngol, 45, 83-89.

van der Steen, M. C., \& Keller, P. E. (2013). The adaptation and anticipation model (ADAM) of sensorimotor synchronization. Front Hum Neurosci, $7,253$.

van Wieringen, A., \& Wouters, J. (2015). What can we expect of normallydeveloping children implanted at a young age with respect to their auditory, linguistic and cognitive skills? Hear Res, 322, 171-179.
Vongpaisal, T., Caruso, D., Yuan, Z. (2016). Dance movements enhance song learning in deaf children with cochlear implants. Front Psychol, 7, 835.

Wang, Y., Shafto, C. L., Houston, D. M. (2018). Attention to speech and spoken language development in deaf children with cochlear implants: A 10-year longitudinal study. Dev Sci, 21, e12677.

Wechsler-Kashi, D., Schwartz, R. G., Cleary, M. (2014). Picture naming and verbal fluency in children with cochlear implants. J Speech Lang Hear Res, 57, 1870-1882.

Wieland, E. A., McAuley, J. D., Dilley, L. C., Chang, S. E. (2015). Evidence for a rhythm perception deficit in children who stutter. Brain Lang, 144, 26-34. 